Regular Article

\title{
Factor XIII improves platelet adhesion to fibrinogen by protein disulfide isomerase-mediated activity
}

\author{
Judith Lahav ${ }^{\text {a }}$, Ariella Tvito ${ }^{\mathrm{a}, \mathrm{b}}$, Zsuzsa Bagoly ${ }^{\mathrm{c}}$, Rima Dardik ${ }^{\mathrm{d}}$, Aida Inbal ${ }^{\mathrm{b}, *}$ \\ ${ }^{a}$ Hemostasis Laboratory, Rabin Medical Center, Beilinson Hospital, Petach Tikva and Sackler Faculty of Medicine, Tel Aviv University, Tel Aviv, Israel \\ b Thrombosis and Hemostasis Unit, Rabin Medical Center, Beilinson Hospital, Petach Tikva and Sackler Faculty of Medicine, Tel Aviv University, Tel Aviv, Israel

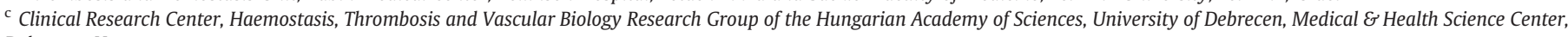 \\ Debrecen, Hungary \\ d Thrombosis and Hemostasis Unit, Sheba Medical Center, Tel Hashomer, Israel
}

\section{A R T I C L E I N F O}

\section{Article history}

Received 20 September 2012

Received in revised form 13 November 2012

Accepted 5 December 2012

Available online 28 December 2012

\section{Keywords:}

Factor XIII

PDI

Transglutaminase

platelet adhesion

\begin{abstract}
A B S T R A C T
Background: Factor XIII (FXIII), a plasma pro-transglutaminase, consists of two A subunits and two B subunits (FXIIIA2B2). Following activation by thrombin, it cross-links fibrin chains at the final step of coagulation. We previously reported that FXIII subunit A (FXIIIA) serves as a protein disulfide isomerase (PDI), and that PDI promotes platelet adhesion and aggregation.

Objective: This study sought to examine possible mechanistic effect of FXIII on platelet adhesion to fibrinogen; specifically, the role of its PDI activity.

Methods: Ex vivo experiments: Blood platelets derived from five patients with hereditary FXIIIA deficiency before and after treatment with Fibrogammin-P (FXIIIA2B2 concentrate) were washed and incubated on immobilized fibrinogen. Bound platelets were stained and counted by microscopy. In vitro experiments: Platelets derived from patients before treatment and five healthy controls were washed and analyzed for adhesion in the presence or absence of Fibrogammin-P or recombinant FXIII (FXIIIA2 concentrate).

Results: In ex vivo experiments, one hour after Fibrogammin-P treatment, mean ( \pm SEM) platelet adhesion to fibrinogen increased by $27 \pm 2.32 \%(\mathrm{p}<0.001)$. In in vitro experiments, treatment with Fibrogammin-P or recombinant FXIII (10 IU/mL each) enhanced platelet adhesion to fibrinogen (in patients, by $29.95 \pm 6.7 \%$ and $29.05 \pm 5.3 \%$, respectively; in controls, by $26.06 \pm 3.24 \%$ and $26.91 \pm 4.72$, respectively; $\mathrm{p}<0.04$ for all). Iodoacetamide-treated FXIII (I-FXIII), where transglutaminase activity is blocked, showed similar enhanced adhesion as untreated FXIII. By contrast, addition of an antibody that specifically blocks FXIIIA-PDI activity inhibited FXIII-mediated platelet adhesion to fibrinogen by $65 \%$.

Conclusion: These findings indicate that FXIII-induced enhancement of platelet adhesion is mediated by FXIII-PDI activity.
\end{abstract}

(c) 2012 Elsevier Ltd. All rights reserved.

\section{Introduction}

Coagulation factor XIII (FXIII) is a plasma pro-transglutaminase. Following activation by thrombin, it cross-links fibrin chains at the final step of coagulation to form a soluble clot [1]. FXIII circulates in plasma as a heterotetramer of two A subunits (FXIIIA2 - the active transglutaminase) and two B subunits (FXIIIB2 - the carrier protein) [1]. Besides its role in hemostasis, FXIII accelerates wound healing [2], probably by its pro-angiogenic effects [3] as well as by stimulation of monocytes and fibroblasts [4]. Several new lines of evidence point

Abbreviations: PDI, protein disulfide isomerase; rFXIII, recombinant FXIII; I-FXIII, iodoacetamide-treated FXIII; FXIIIa, thrombin-activated FXIII; PRP, platelet-rich plasma.

* Corresponding author at: Thrombosis and Hemostasis Unit, Rabin Medical Center, Beilinson Hospital, Petach Tikva 49100, Israel. Tel.: + 9723 9377912; fax: + 9723920 1568 .

E-mail address: aidainbal@hotmail.com (A. Inbal). to the involvement of FXIII in platelet function as well [5-8]. FXIII subunit A (FXIIIA) was detected on thrombin-receptor-activated platelets [5], and platelets were found to adhere to FXIII-covered surface [6]; this interaction depended on the intact fibrinogen binding to integrin $\alpha \operatorname{IIb} \beta 3[5,6]$. Accordingly, no clot retraction was noted in FXIIIA knock-out mice [7], and patients with FXIII deficiency, a rare hereditary life-long bleeding disorder [8], showed reduced fibrinogen binding to thrombin-stimulated platelets and reduced platelet adhesion to fibrinogen-covered surface [9].

We recently reported that FXIIIA has protein disulfide isomerase (PDI) activity, independent of its transglutaminase activity [10]. Cellsurface-localized, membrane-bound PDI is essential for sustaining the binding of fibrinogen, fibronectin, and collagen to platelet integrins $\alpha \operatorname{IIb} \beta 3, \alpha 5 \beta 1$, and $\alpha 2 \beta 1$, respectively [11,12], thereby regulating platelet adhesion [11,12] and aggregation [13]. PDI also regulates the activity of L-selectin, a cell-adhesion molecule found on leukocytes [14]. Specifically, it was found to mediate the entry of human immunodeficiency virus into lymphocytes [15] and the entry of nitric oxide into cells [16]. 
In the present study, on the basis of our earlier findings that PDI plays a mediatory role in platelet adhesion [11-13] and that FXIIIA exerts PDI activity [10], we sought to examine the relative contributions of FXIII PDI or transglutaminase activity to platelet adhesion to fibrinogen.

\section{Materials and Methods}

\section{Materials}

Fibrogammin-P (FXIIIA2B2 concentrate) was purchased from ZLB-Behring, Marburg, Germany. Recombinant FXIII (rFXIII) was a gift from Novo Nordisk, Bagsvaerd, Denmark. Purified FXIII, used to test the inhibitory effect of anti-FXIII antibody, was described previously [10]. Rabbit anti-human factor XIII A-subunit antiserum, (Assera XIII A), was purchased from Diagnostica Stago, Asnieres sur Seine, France. Sheep anti-human factor XIIIA2B2 (SAXIII-IG) was purchased from Affinity Biologicals Inc. Normal rabbit serum and an irrelevant antiserum raised in rabbit (antibiotin), were purchased from Sigma, Israel.

To prepare iodoacetamide-treated FXIII (I-FXIII), approximately $100 \mathrm{IU} / \mathrm{ml}$ reconstituted Fibrogammin $\mathrm{P}$ was activated by thrombin immobilized on Affi-gel-10 beads (Bio-Rad Laboratories, Hercules, CA, USA), as described previously [4]. To generate FXIII with blocked transglutaminase activity (I-FXIII) the thrombin-activated Fibrogammin-P (FXIIIa) was inactivated by treatment with $3 \mathrm{mM}$ iodoacetamide for 30 minutes at $22{ }^{\circ} \mathrm{C}$. Free iodoacetamide was removed by dialysis.

\section{Patients and Controls}

Five patients with hereditary FXIIIA deficiency and five healthy control subjects were studied. None of the control subjects took medication affecting platelet function for two weeks prior to the study. The criteria for diagnosis of FXIIIA deficiency as well as the genotype of the patients were published previously $[16,17]$. The patients were being treated on a prophylactic basis with $20 \mathrm{IU} / \mathrm{kg}$ of Fibrogammin-P every 4 weeks.

\section{Study Design}

The study was divided into two parts. For the ex vivo experiments, we used platelets from blood samples drawn from each patient before and one hour after treatment. For the in vitro experiments, we used platelets from blood samples drawn from the patients before treatment and from the control subjects. The study was approved by the Hospital Ethics Committee and informed consent was obtained from each participant.

\section{Preparation of Platelet-rich Plasma (PRP) and Washed Platelets}

Venous blood from patients and controls was drawn from the antecubital vein following loose application of a tourniquet. The blood was anticoagulated with trisodium citrate $(0.0108 \mathrm{M})$ and processed within 1 hour of collection. PRP was prepared by centrifugation at $700 \mathrm{rpm}$ for $15 \mathrm{~min}$. Washed platelets were prepared as previously described [12]. Briefly 1/10 volume of CTADX10 (citric acid $0.11 \mathrm{M}$, theophylline $15 \mathrm{mM}$, adenosine $3.7 \mathrm{mM}$, and dipyridamole $0.198 \mathrm{mM}$ at $\mathrm{pH}$ 5.0) was added to the PRP followed by centrifugation at $2000 \mathrm{rpm}$ for $10 \mathrm{~min}$. The platelet precipitate was resuspended in saline containing ACD (citric acid $71 \mathrm{mM}$, sodium citrate $0.085 \mathrm{mM}$, D-glucose $11 \mathrm{mM}$ ), centrifuged, and resuspended in adhesion buffer (NaCO3 $12 \mathrm{mM}, \mathrm{NaCl}$ $138 \mathrm{mM}$, D-glucose $5.5 \mathrm{mM}$, KCl $2.9 \mathrm{mM}$, HEPES $50 \mathrm{mM}$, CaCl2 $1 \mathrm{mM}$, $\mathrm{MgCl} 22 \mathrm{mM}, \mathrm{pH} 7.2$ ).

\section{Platelet Adhesion to Protein-covered Plastic}

For the ex vivo experiments, the washed platelets from blood drawn before and one hour after infusion of $20 \mathrm{IU} / \mathrm{kg}$ Fibrogammin-P were incubated on fibrinogen-covered substrata. Adhesion was quantitated by counting the bound cells under light microscopy, as previously described [18]. For the in vitro experiments, the washed platelets from the healthy controls and patients before treatment were analyzed for adhesion in the presence or absence of $10 \mathrm{IU} / \mathrm{mL}$ Fibrogammin-P, or I-FXIII, or rFXIII. In some experiments, antibodies were added $20 \mathrm{~min}$ prior to adhesion at a 1:20 final dilution. In these experiments, PDIinhibiting rabbit anti-FXIIIA antibody, sheep anti FXIIIA2B2 antibody with no inhibitory effect on PDI activity, normal rabbit serum and an irrelevant antiserum raised in rabbit were used at the same dilution as controls. Adhesion was quantitated as previously described [18].

Effect of Anti-FXIIIA Antibodies on Transglutaminase or PDI Activity of FXIII

Highly purified human plasma-derived FXIIIA2B2 was prepared from the pooled plasma of healthy volunteers and thrombin-activated (FXIIIa) according to the procedure of Lorand et al. [19]. In brief, $50 \mu \mathrm{g} / \mathrm{ml}(2.4 \mathrm{U} / \mathrm{ml})$ FXIIIA2B2 in $50 \mathrm{mM}$ HEPES, $100 \mathrm{mM} \mathrm{NaCl}$, and $5 \mathrm{mM} \mathrm{CaCl} 2$ at pH 7.4 was activated by $20 \mathrm{U} / \mathrm{ml}$ human thrombin for $5 \mathrm{~min}$ at $37^{\circ} \mathrm{C}$; thrombin was then blocked by $30 \mathrm{U} / \mathrm{ml}$ hirudin (Sigma-Aldrich, St. Louis, MO). FXIIla was incubated with an equal volume of different antibody dilutions or HEPES- $\mathrm{NaCl}$ buffer for $30 \mathrm{~min}$ at $37{ }^{\circ} \mathrm{C}$. Its transglutaminase activity was measured by the spectrophotometric method of Kárpáti et al. [20]. The results were expressed as a percentage of FXIIla activity in the absence of antibody dilution.

PDI activity was determined by measuring the ability to restore RNase activity of rdRNase as previously described (10). FXIIIA2B2 was incubated with equal volume of different antibody dilutions or HEPES$\mathrm{NaCl}$ buffer for $30 \mathrm{~min}$ at $37{ }^{\circ} \mathrm{C}$ and PDI activity of FXIII was measured as above.

\section{Statistical Analysis}

T-test was used to compare mean $( \pm$ SEM) platelet adhesion before and after treatment with FXIII concentrate and to compare mean $( \pm$ SEM) platelet adhesion in the presence or absence of different FXIII preparations. $\mathrm{P}<0.05$ was considered significant.

\section{Results}

The ex vivo findings shown in Fig. 1 represent the summation of 56 treatments in five patients with hereditary FXIIIA deficiency. One hour after Fibrogammin-P infusion, platelet adhesion to fibrinogen increased by a $27 \pm 2.32 \%$ from the pretreatment level $(p<0.001)$.

Results of the in vitro experiments are presented in Fig. 2. The addition of Fibrogammin-P $(10 \mathrm{IU} / \mathrm{mL})$ to the washed platelets from the patients enhanced adhesion to fibrinogen by $29.95 \pm 6.7 \%$, and the addition of rFXIII $(101 \mathrm{U} / \mathrm{mL})$ enhanced adhesion by $29.05 \pm 5.3 \%$ ( $n \geq 20, p<0.04$ for each). Similar results were obtained with washed control platelets $(26.06 \pm 3.24 \%$ and $26.91 \pm 4.72 \%$, respectively, $\mathrm{n} \geq 10$; $\mathrm{p}<0.04$ for each) (Fig. 2).

To distinguish between the transglutaminase and PDI activity of FXIII, we used a specific rabbit anti-FXIIIA antibody which inhibited PDI activity in our previous study [10]. The addition of anti-FXIIIA antibody (at a dilution of $1: 20$ ) to plasma-purified FXIII inhibited $75 \%$ of FXIIIA PDI activity without inhibiting transglutaminase activity (Fig. 3).

The comparison of the effect of Fibrogammin-P alone to that of anti-FXIIIA antibody + Fibrogammin-P on patients' platelet adhesion is shown in Fig. 4. Relative to the untreated platelets, the addition of Fibrogammin-P enhanced platelet adhesion to fibrinogen by approximately $30 \%$. The combination of rabbit anti-human FXIIIA antibody known to inhibit PDI activity [10] with Fibrogammin-P resulted in a $65 \%$ reduction in adhesion (Fig. $4 ; \mathrm{n}=10 ; \mathrm{p}<0.001$ ), which is beyond the expected $30 \%$ inhibition of the effect of Fibrogammin $(n=10$, $\mathrm{p}<0.05$; Fig. 4). This finding may indicate that this anti-FXIIIA 


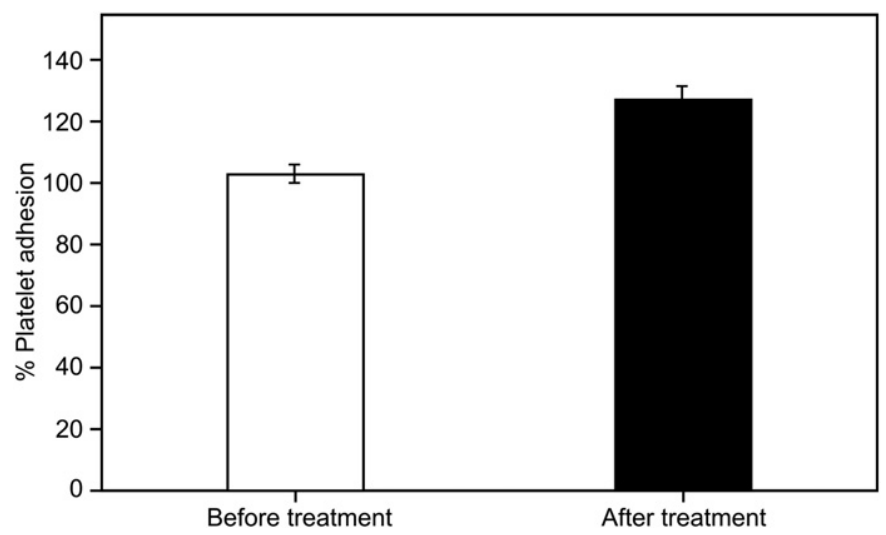

Fig. 1. Fibrinogen adhesion of platelets derived from patients with hereditary FXIIIA deficiency before and after treatment with Fibrogammin-P. Washed platelets were prepared from patients' PRP before and one hour after infusion of $20 \mathrm{IU} / \mathrm{kg}$ Fibrogammin and incubated on fibrinogen-covered surface. Bound platelets were counted by microscopy. The results are expressed as mean \pm SEM percent increase in adhesion of 56 treatments. Each experiment during one treatment was done in quadruplicate.

antibody inhibits PDI activity of untreated platelets. Indeed, addition of this antibody to untreated platelets inhibited $36 \pm 11.6 \%$ of platelet adhesion to fibrinogen $(p<0.01$; data not shown). By contrast to the rabbit anti-FXIIIA antibody we found that the sheep anti-FXIII A2B2 antibody showed no inhibition of PDI activity. Addition of this sheep anti-human FXIIIA2B2 antibody, had no effect on FibrogamminP-mediated enhancement of platelet adhesion: $26 \% \pm 18 \%$ increase in adhesion in the presence and $22 \% \pm 9 \%$ increase in adhesion in the absence of the antibody $(p=0.6)$.

Incubation of the patients' platelets with I-FXIII (10 IU/ml), in which transglutaminase activity is blocked by iodoacetamide, increased platelet adhesion by $27.6 \pm 2.1 \%(n=8)$, similar to the increase observed with Fibrogammin-P ( $33 \pm 6.7 \%$, Fig. $4 ; n=19 ; p=0.8)$. This finding suggests that transglutaminase activity of FXIII does not mediate increased platelet adhesion to fibrinogen.

\section{Discussion}

The involvement of FXIII transglutaminase activity in platelet spreading was suggested by previous findings that monodansylcadaverine, in the absence of added FXIII, diminished filopodia formation of normal

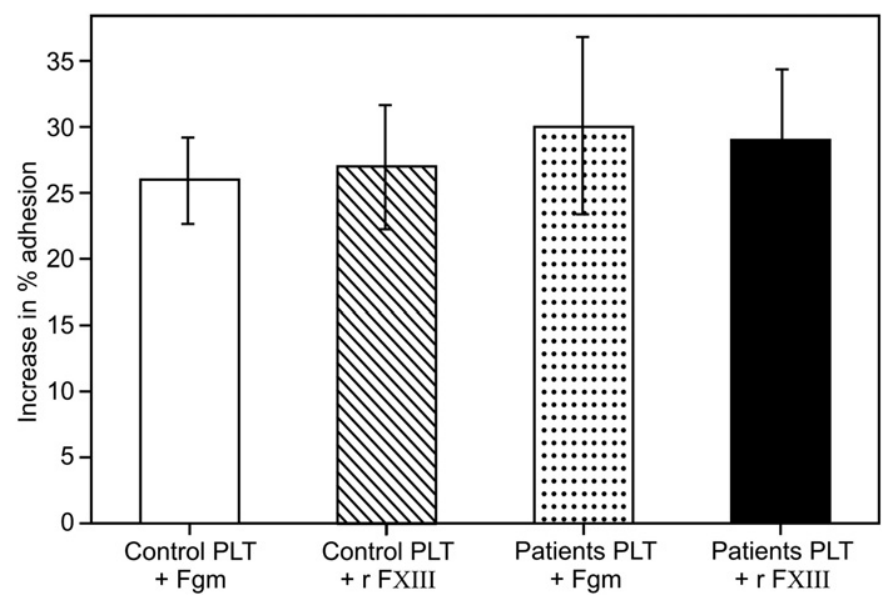

Fig. 2. Enhanced platelet adhesion to fibrinogen following in vitro addition of FXIIIA2B2 or FXIIIA2. Washed platelets obtained from the patients prior to treatment or from normal control subjects were incubated on fibrinogen-covered surface in the presence or absence of $10 \mathrm{IU} / \mathrm{mL}$ of either Fibrogammin-P (FXIIIA2B2) or rFXIII (FXIIIA2). Bound platelets were counted by microscopy. Each experiment was performed in quadruplicate. The results are expressed as mean \pm SEM percent platelet adhesion in the presence of added FXIII relative to the adhesion observed before addition of FXIII.

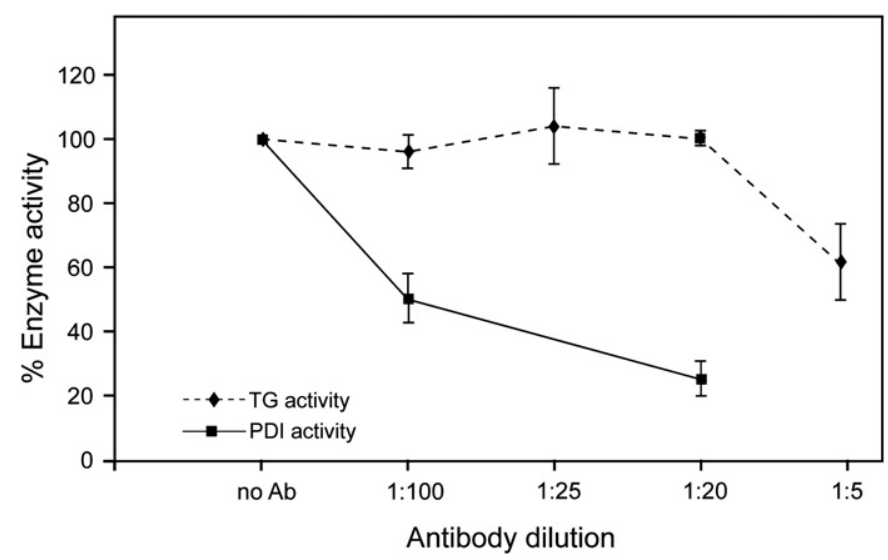

Fig. 3. Anti-FXIII antibody inhibition of FXIII-PDI activity but not FXIII - transglutaminase activity. Serial dilutions of anti-human factor XIII A-subunit antibody were incubated with plasma-purified FXIII and either transglutaminase or PDI activity was determined. Results are expressed as mean $\pm \operatorname{SEM}(\mathrm{n}=3)$.

platelets on fibrinogen [9] and that clot retraction in mice was inhibited by the FXIII transglutaminase inhibitor, cystamine [7]. Given our earlier finding that FXIIIA has PDI activity which is independent of its transglutaminase activity [10], we examined the relative roles of the two activities in platelet adhesion to fibrinogen.

We show, for the first time, that in patients with hereditary FXIIIA deficiency, treatment with FXIIIA2B2 (Fibrogammin-P) increases platelet adhesion to fibrinogen by almost $30 \%$ (Fig. 1). These findings are in accordance with previous reports that platelets from FXIII-deficient patients exhibit reduced adhesion to fibrinogen in vitro [9]. Furthermore, the in vitro addition of unactivated FXIIIA2B2 (Fibrogammin-P) or unactivated FXIIIA2 (rFXIIIA), neither of which exhibits transglutaminase activity, to either the patient or control platelets, enhanced platelet adhesion to fibrinogen (Fig. 2), suggesting that transglutaminase activity is not necessary for platelet adhesion to fibrinogen. This conclusion was further supported by our finding that adhesion of the patients' platelets to fibrinogen in the presence of FXIII treated with iodoacetamide, in which transglutaminase activity is blocked, was indistinguishable from that of the patient's platelets in the presence of unactivated FXIII (Fig. 4). Thus, in our hands transglutaminase activity did not mediate the enhanced adhesion of platelets to fibrinogen.

Our earlier work showed that unactivated FXIIIA2B2, I-FXIII, and rFXIIIA2 all have similar PDI activity [10], pointing to a possible role

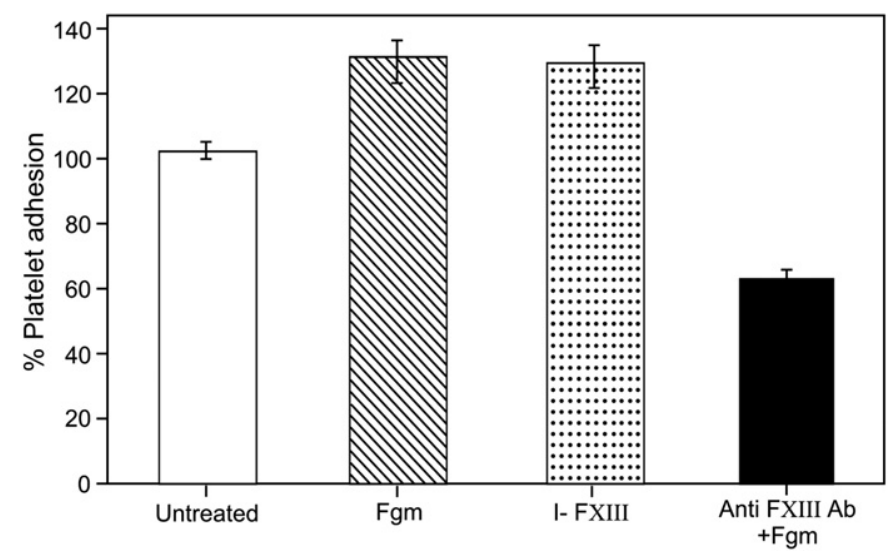

Fig. 4. Enhanced platelet adhesion in the presence of I-FXIII and reduced platelet adhesion by anti-FXIII antibody. Washed platelets obtained from the patients before treatment were incubated on fibrinogen-covered surface in the presence or absence of either $10 \mathrm{IU} / \mathrm{mL}$ I-FXIII or anti-FXIIIA antibody at 1:20 dilution. Bound platelets were counted by microscopy. Each experiment was performed in quadruplicate. Results are expressed as mean \pm SEM percent platelet adhesion in the presence of I-FXIII $(n=8)$ or anti-FXIII antibody relative to their absence. 
for FXIII PDI in platelet adhesion. Accordingly, in the present study, the addition of rabbit anti-FXIIIA antibody that interferes with FXIIIA PDI activity but not FXIIIA transglutaminase activity (Fig. 3 ) reduced the adhesion of the platelets in the presence of FXIII concentrate (Fig. 4), while sheep anti-FXIIIA2B2 antibody that does not interfere with PDI activity had no effect on adhesion. Moreover, the anti-PDI activity of rabbit anti FXIIA antibody reduced the adhesion of untreated platelets, probably by inhibiting surface-associated PDI on the platelets which is required for intact platelet adhesion $(11,12)$.

By contrast, Jayo et al. [9] and Kasahara et al. [7] reported that monodansylcadaverine and cystamine, competitive donors for transglutaminase, inhibited FXIII-mediated adhesion. The reason for the discrepancy from our study is not clear. If it stems from differences in the experimental procedures, it is possible that both the transglutaminase and PDI activities of FXIII support platelet adhesion to fibrinogen in an additive manner. Alternatively, it is possible that the inhibitors used to block transglutaminase activity in the earlier studies $[7,9]$ were not specific. Both monodansylcadaverine and cystamine may block other thiol-dependent enzymes, particularly calmudulin [21]. Indeed, one study showed that caspase-3 is inhibited by cystamine [22]. Cystamine forms mixed disulfides with PDI, and in the cellular setting, it might inhibit the PDI activity of FXIII as well as its transglutaminase activity. Our use of an antibody that specifically blocks only the PDI activity of FXIII is a more direct means to address this question.

Recently, researchers found that platelets adhered to FXIII-covered surface independently of FXIII transglutaminase or PDI activity [6]. The independence of transglutaminase activity is in line with our previous results [10] and the results of the present study. These data also confirm our previous observation that FXIII can bind directly to platelets [23]. However, in another study, we showed that PDI-dependent platelet adhesion is specific to platelet integrin-mediated adhesion [12,13]. Thus, the addition of FXIII following established platelet integrin-fibrinogen interaction, as in the present work, leaves FXIIIA PDI activity free to enhance this interaction.

The increase in platelet adhesion elicited by unactivated FXIIIA2B2 or rFXIIIA2, as well as by I-FXIII, and the inhibition of adhesion by PDIblocking antibody, supports the hypothesis that the enhancement of platelet adhesion associated with FXIII is mediated by its PDI activity. This may have important therapeutic implications for the use of FXIII concentrates. In addition to their well-established effect on secondary hemostasis (clot stability), FXIII concentrates might also enhance primary hemostasis (platelet function) in patients with hereditary FXIIIA deficiency.

\section{Conflict of Interest Statement}

None of the authors has a direct or indirect proprietary interest in the manuscript.

\section{Funding Source}

This work was supported in part by grants from the Chief Scientist's Office of the Ministry of Health, Israel.

\section{References}

[1] Muszbek L, Yee VC, Hevessy Z. Blood coagulation factor XIII: structure and function. Thromb Res 1999;94:271-305.

[2] Inbal A, Lubetsky A, Krapp T, Castel D, Shaish A, Dickneitte G, et al. Impaired wound healing in factor XIII deficient mice. Thromb Haemost 2005;94:432-7.

[3] Dardik R, Solomon A, Loscalzo J, Eskaraev R, Bialik A, Goldberg F, et al. Novel proangiogenic effect of factor XIII associated with suppression of thrombospondin 1 expression. Arterioscler Thromb Vasc Biol 2003;23:1472-7.

[4] Dardik R, Krapp T, Rosental E, Loscalzo J, Inbal A. Effect of Factor XIII on monocyte and fibroblast function. Cell Physiol Biochem 2007;19:113-20.

[5] Nagy B, Simon Z, Bagoly Z, Muszbek L, Kappelmayer J. Binding of plasma factor XIII to thrombin-receptor activated human platelets. Thromb Haemost 2009;101:83-9.

[6] Magwenzi SG, Ajjan RA, Stendeven KF, Parapia LA, Naseem KM. Factor FXIII supports platelet activation and enhances thrombus formation by matrix proteins under flow conditions. J Thromb Haemost 2011;9:820-33.

[7] Kasahara K, Souri M, Kaneda M, Miki T, Yamamoto N, Ichinose A. Impaired clot retraction in factor XIII-deficient mice. Blood 2010;115:1277-9.

[8] Inbal A, Yee VC, Kornbrot N, Zivelin A, Brenner B, Seligshon U. Factor XIII deficiency due to a Leu660Pro mutation in the factor XIII subunit-A gene in three unrelated Palestinian Arab families. Thromb Haemost 1977;77:1062-7.

[9] Jayo A, Conde I, Lastres P, Jimenez-Yuste V, Gonzalez-Manchon C. New insights into the expression and role of platelet factor XIII-A. J Thromb Haemost 2009;7: 1184-91.

[10] Lahav J, Karniel E, Bagoly Z, Sheptovitsly V, Dackik R, Inbal A. Coagulation factor XIII serves as a protein disulfide isomerase. Thromb Haemost 2009;101:840-4.

[11] Lahav J, Gofer-Dadosh N, Luboshitz J, Hess O, Shaklai M. Protein disulfide isomerase mediates integrin-dependent adhesion. FEBS Lett 2000;475:89-92.

[12] Lahav J, Wijnen EM, Hess O, Hamaia SW, Griffiths D, Makris M, et al. Enzymatically catalyzed disulfide exchange is required for platelet adhesion to collagen via integrin alpha2beta1. Blood 2003;102(6):2085-92.

[13] Lahav J, Jurk K, Hess O, Barnes MJ, Farndale RW, Lubositz J, et al. Sustained integrin ligation involves extracellular free sulfhydryls and enzymatically catalyzed disulfide exchange. Blood 2002;100(7):2472-8.

[14] Bennett TA, Edwards BS, Sklar LA, Rogelis S. Sulfhydryl regulation of L-selectin shedding: Phenylarsine oxide promotes activation-independent L-selectin shedding from leukocytes. J Immunol 2000;164:4120-9.

[15] Gallina A, Hanley TM, Mandel R, Trahey M, Broder CC, Viglinati GA et al. Inhibitors of protein disulfide isomerase prevent cleavage of disulfide bonds in receptor-bound Glycoprotein 120 and prevent HIV-1 entry. J Biol Chem 2002;277:50579-88.

[16] Zai A, Rudd MA, Scribner AW, Loscalzo J. Cell-surface protein disulfide isomerase catalyzes transnitrosation and regulates intracellular transfer of nitric oxide. J Clin Invest 1999;103:393-9.

[17] Vysokovsky A, Saxena R, Landau M, Zivelin A, Eskaraev R, Rosenberg N, et al. Seven novel mutations in the Factor XIII A-subunit gene causing hereditary Factor XIII deficiency in ten unrelated families. J Thromb Haemost 2004;2:1790-7.

[18] Lahav J. Thrombospondin inhibits adhesion of platelets to glass and proteincovered substrata. Blood 1988;71:1096-9.

[19] Lorand L, Credo RB, Janus TG. Factor XIII (fibrin stabilizing factor). Methods Enzymol 1981;80:333-41.

[20] Kárpáti L, Penke B, Katona E, Balogh I, Yamosi G, Muszbek L. A modified, optimized kinetic photometric assay for the determination of blood coagulation factor XIII activity in plasma. Clin Chem 2000;46:1946-55.

[21] Lorand L. DRPLA aggregation and transglutaminase, revisited. Nat Genet 1998;20: 231.

[22] Lesort M, Lee M, Tucholski J, Johnson GY. Cystamine inhibits caspase activity, implications for the treatment of polyglutamine disorders. J Biol Chem 2003;278: 3825-30.

[23] Dardik R, Shenkman B, Tamarin I, Eskaraev R, Harsfalvi J, Varon D, et al. Factor XIII mediates adhesion of platelets to endothelial cells through $\alpha_{v} \beta_{3}$ and glycoprotein IIb/IIla integrins. Thromb Res 2002;105:317-23. 